\title{
Robustness of computational time reversal imaging in media with elastic constant uncertainties
}

\author{
M. Scalerandi, ${ }^{1}$ M. Griffa, ${ }^{2, a)}$ and P. A. Johnson ${ }^{3}$ \\ ${ }^{1}$ Department of Physics, Politecnico di Torino, C.so Duca degli Abruzzi 24, 10129 Torino, Italy \\ ${ }^{2}$ Laboratory for Building Science and Technology, Swiss Federal Laboratories for Materials Testing and \\ Research (EMPA), Dübendorf 8600, Switzerland \\ ${ }^{3}$ Geophysics Group (EES-17), Los Alamos National Laboratory, MS D443, Los Alamos, New Mexico, 87545 \\ USA
}

(Received 31 July 2009; accepted 5 November 2009; published online 11 December 2009)

\begin{abstract}
In order to image a source or a scatterer embedded in a three dimensional solid, acoustic/elastic wave data from an actual experiment are time reversed and backpropagated through a numerical model of the medium. The model makes use of estimates for the elastic constants of the laboratory solid. These estimates may not be very precise, for example, due to experimental uncertainties. Poor characterization of the medium leads to the degradation of the time reversal focus, therefore, to poor medium imaging. In this work, we report on the results of investigating the time reversal focus degradation as the estimates depart from the real values. Very small deviations from the medium's actual elastic constants degrade the time reversal focus dramatically. However, decreasing the total duration of the signals used for time reversal can attenuate the degradation in some cases. We propose a new method to compensate for the deviations of the model medium's elastic constants from the actual values. Finally, we explore the effects of scatterers that may exist in the laboratory medium, but are not included in the model medium, and show that their presence does not produce significant effects on the time reversal focus. (C) 2009 American Institute of Physics.
\end{abstract}

[doi:10.1063/1.3269718]

\section{INTRODUCTION}

Time reversal (TR) acoustics (TRA) ${ }^{1-3}$ provides the means for locating, in space and time, sources, either active or passive, i.e., scatterers, of elastic and electromagnetic waves. These techniques rely on the basic procedure of TRA, termed the time reversal process (TRP). ${ }^{4}$ In the process, a source, in or adjacent to a medium, generates a wavefield [forward propagation (FP) stage]. A set of sensors (from one to an array of $N$, where $N$ may be large ${ }^{1}$ ) records the signals due to the propagated wavefields. These signals are then time reversed and rebroadcast from the same sensors, now acting as sources [TR backward propagation (TRBP) stage]. When the medium is lossless, the wave equation is invariant for temporal inversion. When the spatial reciprocity principle holds, the rebroadcast wavefields focus at the original source(s) location(s), approximately reconstructing the original source disturbance. TRP also remains valid in the case of weakly attenuative media, satisfying the spatial reciprocity principle. ${ }^{4}$

TR can be exploited for imaging purposes if the spatiotemporal evolution of the TRBP wavefields is recorded. An imaging condition, i.e., the procedure for constructing the image of the source, is then applied to create the source image.

A similar approach has been developed and used in the field of geophysical prospection (seismic imaging) for more than 30 years, under the name of reverse time migration

\footnotetext{
${ }^{a)}$ Author to whom correspondence should be addressed. Electronic mail: michele.griffa@empa.ch.
}

(RTM). ${ }^{6,7}$ There is a significant difference between RTM and the imaging procedure exploiting the TRP: in the former, both the FP and TRBP wavefields need to be recorded, not only the TRBP one. Indeed, the RTM imaging condition, in the basic form, consists of calculating, at each location of the spatial sampling grid, the temporal cross correlation of the FP and TRBP wavefields. On the contrary, imaging methods in TRA usually require only the TRBP wavefields.

Many TRA imaging techniques have been developed and deployed for practical applications. For example, using ultrasound for imaging scatterers, either linear, such as inhomogeneities or voids, ${ }^{8,9}$ or elastically nonlinear, such as microcracks. ${ }^{10-14}$ Other applications are in the fields of seismology (locating earthquake sources and source characterization), ${ }^{15,16}$ ocean acoustics (locating targets), ${ }^{17}$ and microwave imaging. ${ }^{18,19}$ Some of these techniques address specific problems in locating active source(s)/ scatterer(s), such as to selectively find the position of a source in the presence of an unknown number of sources, 8,20 including the case of weak sources masked by stronger ones. $^{21,22}$

The need to record the spatial/temporal evolution of one TRBP wavefield is a potential limitation for imaging by TRA. For example, in nondestructive evaluation applications, the wavefields can normally be measured only on the surface of the specimen so that the imaging is restricted to the surface. When the active source(s) or scatterer(s) is(are) embedded in the bulk of a three dimensional (3D) volume (which is almost always the case in the geophysical context), a viable alternative consists of a computational method: ${ }^{23}$ the TRBP stage is performed via a numerical simulation, adopt- 
ing a realistic model of the propagation medium and using the experimentally recorded FP signals as time-evolving boundary conditions. In RTM, also the FP stage needs to be simulated numerically using a realistic model of the illumination source. ${ }^{7}$

The success of this approach, exploiting numerical simulations instead of laboratory/field measurements, relies on the following three fundamental assumptions:

- the model used for the simulation takes into account the complexity of the structure of the medium and of the wave propagation process;

- the sources of the TRBP simulations are modeled properly; and

- the physical parameters (elastic moduli, density, boundary conditions, etc.) of the simulated medium approximately match the actual physical properties.

The first issue does not constitute a significant problem. Existing wave propagation models can take into account complex rheologies and structured geometries. ${ }^{24}$ The second issue is not as important as it may appear due to the robustness of the TRP provided the phases of the signals are accounted for. ${ }^{25}$ The third issue, however, is crucial.

The mismatch between the real and the model media is comprised of two fundamental aspects: the boundary conditions and the physical parameters (moduli and density). The medium physical parameters can be only estimated with a certain level of uncertainty. The influence of such uncertainty has been experimentally investigated in the context of studying the performance of the TRP in the presence of timeevolving random media. For instance, Tourin et al. investigated the amount of perturbation of the TR focus, in water and in the presence of a random distribution of rigid pointlike scatterers, ${ }^{26}$ as a consequence of varying the speed of sound between the FP and TRBP stages, changing the water temperature, or varying the scatterer distribution configuration (different realization of the random medium). ${ }^{27} \mathrm{Kim}$ et al. investigated the robustness of the TRP in an ocean waveguide, whose sound speed vertical profile changes in time due to internal waves. ${ }^{28}$ Liu et al. performed similar studies in the case of imaging targets or sources embedded in clutter using the TRP with electromagnetic waves, ${ }^{29}$ while Bal and Verastegui $^{30}$ investigated theoretically the robustness of the TRP to medium mismatching considering highly multiple scattering regimes. In all cases, the robustness of the TRP was extremely weakened by perturbations in the properties of the medium. Indeed, as proven by several studies in the field of coda wave interferometry, ${ }^{31}$ multipathing in a medium increases the dependence of the wavefields on the medium structure, and a small change in the propagation medium implies a significant change in the signals recorded at a certain location due to the propagation. In the field of seismic imaging, this medium mismatch issue has been the object of an intense and thorough investigation. ${ }^{32}$ RTM is highly affected by medium mismatch because of the use of numerical simulation for estimating both the FP and TRBP wavefields. The extreme sensitivity of wave propagation to medium mismatch in the presence of highly heterogeneous media, as the Earth crust is, has been exploited not only for imaging their

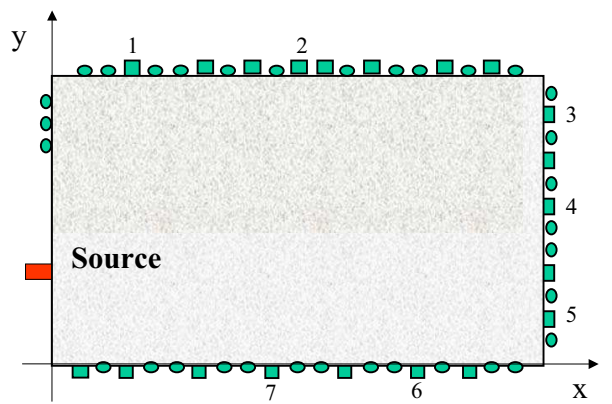

(a)

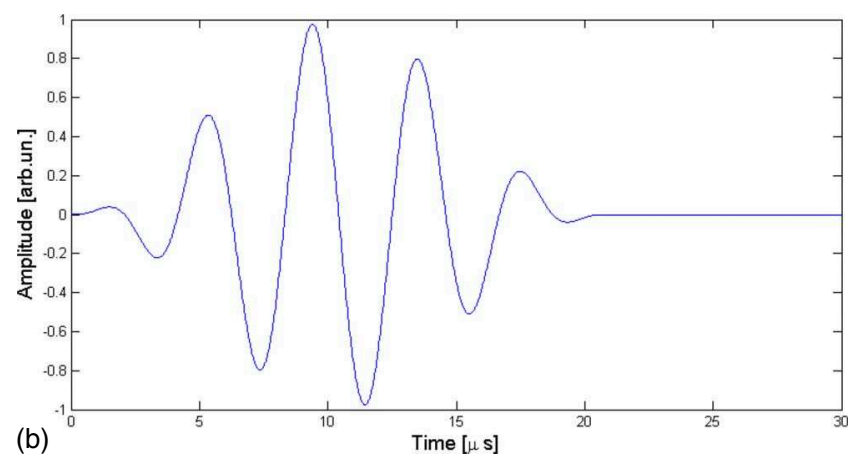

FIG. 1. (Color online) (a) Schematic representation of the 2D sample used for the case study. The sample is an aluminum plate $\left(10 \times 6 \mathrm{~cm}^{2}\right)$. The rectangle represents the source position, while squares and circles are located at the receivers positions. (b) Source signal corresponding to a force applied at the source position and oriented in the $X$ direction.

structure but also for solving inverse problems, i.e., for estimating the medium's physical parameters such as wave velocity and mass volumetric density. ${ }^{33}$

In this work, we specifically investigate two sources of inaccuracies in modeling:

- the elastic constants of the modeled medium for the TRBP simulation do not match those of the real medium, but both media are homogeneous (Sec. III) and

- the model medium is assumed homogeneous, while that is not the case for the real medium, which is, more likely, a random medium or it might contain small, hidden inclusions (Sec. IV).

In both cases, we analyze the robustness of TRA imaging approaches, exploiting the numerical simulation of the TRBP. In Sec. V, we provide some suggestions for improving the robustness of the TRP to medium perturbations and uncertainties.

\section{NUMERICAL TR}

\section{A. The case study}

We created a model of a two dimensional (2D) sample, as shown in Fig. 1(a), in order to perform our analysis. Due to the large number of simulations required, 3D calculations were not extensively conducted; however, we performed a few similar tests in 3D (not reported here for brevity) in order to check for consistency of the results obtained in 2D. The sample is a rectangular $\left(10.0 \times 6.0 \mathrm{~cm}^{2}\right)$ aluminum (elastic isotropic) sample, with longitudinal wave velocity $v_{L}=6498 \mathrm{~m} \mathrm{~s}^{-1}$, shear wave velocity $v_{S}=4346 \mathrm{~m} \mathrm{~s}^{-1}$, and 
density $\rho=2700 \mathrm{~kg} \mathrm{~m}^{-3}$. We neglected the modeling of the viscoelastic properties, giving rise to attenuation.

We placed a source at a position $\mathbf{r}_{0}=(0.0,2.0) \mathrm{cm}$. This source applied a forcing in the form of a sine signal modulated in amplitude by a squared sine signal [see Fig. 1(b)],

$$
f\left(\mathbf{r}_{0}, t\right)=\sin (2 \pi \nu t) \sin ^{2}(\pi t / \zeta)
$$

The source, in general, a vector, in this case was assumed to be a force parallel to the $X$ direction. The central frequency was $\nu=240 \mathrm{kHz}$ and the source duration was $\zeta=20.8 \mu \mathrm{s}$. The central frequency corresponds to a longitudinal central wavelength of about $27 \mathrm{~mm}$ and the source signal contains about 5 cycles.

Fifty three pointlike receivers were located on the sample boundaries, as indicated in Fig. 1(a). They were equally spaced (5 mm apart) and recorded a maximum $1.3 \mathrm{~ms}$ long displacement vector signal. The two components (along the $X$ and $Y$ directions) were recorded independently. Finally, we assumed free boundary conditions at the four boundaries of the sample [except for the source position(s)].

\section{B. Numerical simulation of elastic wave propagation}

For the numerical simulation of elastic wave propagation, we used a 2D full displacement explicit FDTD code, based on the local interaction simulation approach (LISA)spring model. ${ }^{34}$ The LISA-spring model was originally developed for simulating elastic wave propagation in highly heterogeneous materials, containing high density of interfaces. It was then expanded in order to take into account nonclassical (nonequilibrium) nonlinear elastic behaviors due to volumetric distribution of microcracks ${ }^{34}$ and/or localized macroscopic ones. The validation of the model along with a comparison to theoretical expectations and real data has been reported elsewhere. ${ }^{35,36}$

In our case, the modeled specimen (homogeneous) was discretized into a $500 \times 300$-node uniform structured Cartesian grid, corresponding to a mesh step $\varepsilon=0.2 \mathrm{~mm}$ along each axis. This FDTD scheme must satisfy convergence and stability conditions. For the case considered, the choice of $\varepsilon$ ensured that each central wavelength of the wavefield was described by a sufficiently large number of points (about 140). The choice of a temporal step $\tau=0.025 \mu$ s satisfies the Courant-Friedrichs-Lewy condition.

\section{Computational imaging using the TRP}

For the imaging technique based on TRA and numerical simulation, the FP, is expected to be performed experimentally, i.e., the receivers record the signals generated by the wavefields propagated in a real-world specimen. Then, these signals are time reversed and used as time-varying boundary conditions for the backward propagation, performed numerically in a simulated medium ideally the same as the real one. In this work, we tested this technique in locating an active source and reconstructing it. We performed the FP step also using a numerical simulation, instead of using the experimentally acquired signals, in order to be able to isolate the effects of the uncertainties in the elastic constants values.
For the back propagation stage, two parameters must be fixed: the number $n$ of receivers recording the signals (the number of TR mirror elements) and the length $\Delta$ of these signals used as input for the back propagation. In order to illustrate general behaviors, we considered three values of $n$ $(n=53,20$, and 7$)$ and three values of $\Delta(\Delta=1.2,0.6$, and $0.1 \mathrm{~ms})$. The three $\Delta$ cases will be called, from now on, long, medium, and short coda, respectively. The term "coda" refers to long lasting signals due to reverberation or multiple scattering. In the $n=20$ case, only transducers denoted with a square in Fig. 1(a) are used. In the $n=7$ case, only transducers labeled with a number in Fig. 1(a) are used.

The computational imaging approach based on the TRP consists of the following steps.

(i) Let us call $\mathbf{u}_{i}(t)$ the vector displacement signal recorded by the receiver $i$ during the FP, $(i=1, \ldots, n)$. Again, these signals were computed in this work via a numerical simulation, in general, they are measured during experiments. Also, we note that these signals are two-component vectors, even though, as shown elsewhere, ${ }^{37}$ the TR procedure works well even with only one component of the signal.

(ii) The reversed signals were generated as $\mathbf{u}_{i}^{\mathrm{rev}}(t)=\mathbf{u}_{i}(\Delta$ $-t)(0<t<\Delta ; i=1, \ldots, n)$; and

(iii) Backward propagation simulations were then performed using the receivers as transducers injecting the full vector signals $\mathbf{u}_{i}^{\text {rev }}(t)$, after conversion into accelerations in order to be applied as forcing signals. Both vector components of the converted versions of $\mathbf{u}_{i}^{\text {rev }}(t)$ were injected. In what follows, we will, in general, indicate with $\mathbf{g}_{\mathbf{r}}$ a vector wavefield, with the dimension of a force, recorded during the backward propagation at a specific spatial location $\mathbf{r}$.

Full displacement numerical simulations provide the means to calculate whatever wavefield is required (velocity, strain, and stress) at all positions in space and time. Three quantities are of particular interest in the analysis of the robustness of the TR approach:

- the signal (force) recorded during the back propagation at the position of the original source as a function of time: $g_{s}(t)$. Again, this is a vector, but in our analysis we concentrated only on its $X$-component, $g_{s}(t)$. Indeed, being the original source signal parallel to the $X$-direction, the $Y$ - component at the focal position is always negligible. $g_{s}(t)$ provides information about the temporal compression of the time reversed signal. In a perfect TR experiment, $g_{s}(t)=f\left(\mathbf{r}_{0}, \Delta-t\right)$;

- the stress field at the time of focusing, i.e., the spatial distribution of the stresses in the sample. We will not analyse this quantity in this paper; and

- the maximum-in-time acceleration (or stress or displacement) field distribution in the sample, i.e., the matrix defined by the maximum value reached by the acceleration (stress and displacement) at each spatial location: 

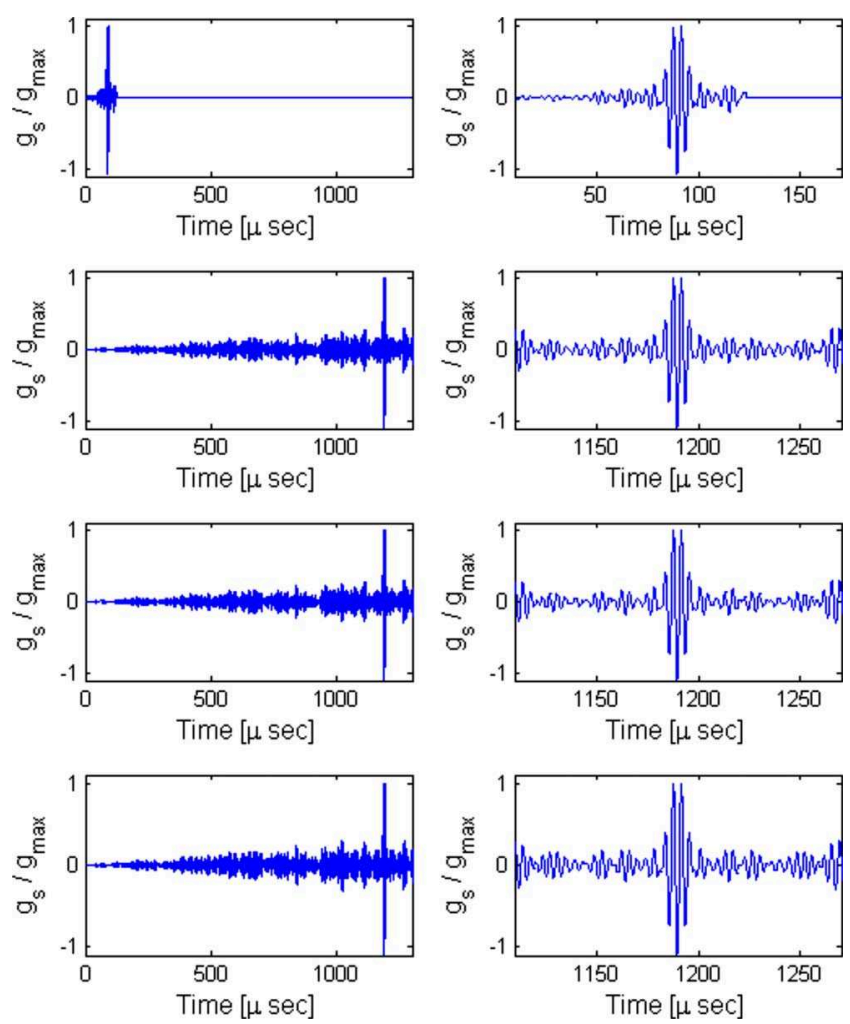

FIG. 2. (Color online) Left column: temporal focusing of the time reversed wavefield at the FP source position for different choices of the parameters $n$ and $\Delta$, respectively, the number of sensors for the TRP and the temporal length of the signal portion used in the time inversion. The wavefield is normalized to its maximum value in time $\left(g_{\max }\right)$. First row: $n=53, \Delta$ $=0.1 \mathrm{~ms}$. Second row: $n=53, \Delta=1.2 \mathrm{~ms}$. Third row: $n=20, \Delta=1.2 \mathrm{~ms}$. Fourth row: $n=7, \Delta=1.2 \mathrm{~ms}$. The backpropagation medium perfectly matches the real medium (FP). The right column provides a zoom around the retrofocal time to appreciate the quality of the reconstruction of the source signal shape [compared with Fig. 1(b)].

$$
M(i, j)=\Lambda \max \left(\left|\mathbf{g}_{i j(i)}\right|\right)
$$

Here $\left|\mathbf{g}_{i j(t)}\right|$ is the norm of the force vector, computed during the backward propagation, at node $(i, j)$ and time $t$ and the maximum is calculated in the whole time interval of the simulation. $\Lambda$ is a renormalization constant from force to acceleration. The matrix $M$ gives information about the spatial focusing of the signal.

In Fig. 2 (left column), we analyze the quality of the temporal focusing for different choices of $n$ and $\Delta$. From the top to the bottom, the following cases are considered: $n$ $=53, \Delta=0.1 \mathrm{~ms} ; n=53, \Delta=1.2 \mathrm{~ms} ; n=20, \Delta=1.2 \mathrm{~ms}$; and $n=7, \Delta=1.2 \mathrm{~ms}$. In all cases, the temporal compression is good and the signal reconstruction excellent (a zoom around the focal time is reported in the right column). Also spatial retrofocalization, for the same cases, is remarkable (see Fig. 3 ). The size of the spot is approximately $\lambda / 4$, in agreement with the diffraction limit. Finally, we mention that, as expected, results for a short coda with $n=7$ are not as good as those reported here due to the apparent decrease in virtual sources corresponding to decreasing the coda length.

We can conclude this section remarking that, as expected, the computational TRA imaging procedure is very robust in locating the active source, independently from the
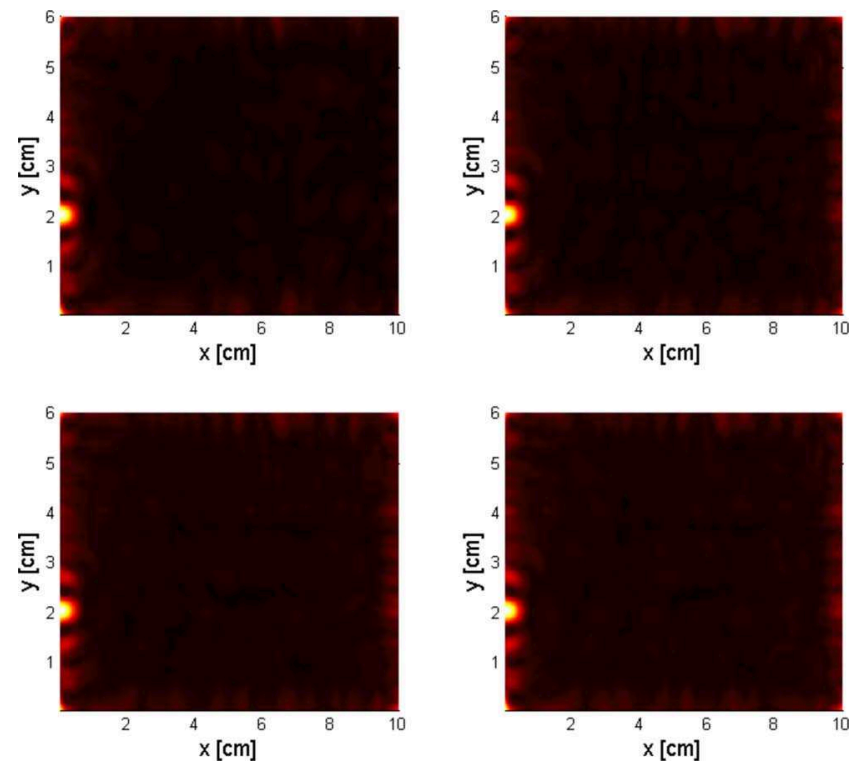

FIG. 3. (Color online) Spatial retrofocusing of the backpropagating wavefield onto the original source location for different choices of the parameters $n$ and $\Delta$, respectively, the number of sensors for the TRP and the length of the signal portion used in the time inversion. The backpropagation medium perfectly matches the real medium (FP). The plots report the matrix $M$ (maximum-in-time acceleration field) defined in Eq. (2). Upper left: $n=53$, $\Delta=0.1 \mathrm{~ms}$. Upper right: $n=53, \Delta=1.2 \mathrm{~ms}$. Lower left: $n=20, \Delta=1.2 \mathrm{~ms}$. Lower right: $n=7, \Delta=1.2 \mathrm{~ms}$.

number of transducers and coda length (within limits), provided the sample used in the simulations matches perfectly the "real" sample (the one used to simulate the FP).

\section{ROBUSTNESS AND UNCERTAINTIES IN THE ELASTIC CONSTANTS}

\section{A. Robustness of the approach}

Here, we address the importance of using the correct physical properties in the model. Our approach is to fix the elastic constants $S_{i j k l}^{\prime}$ of the TRBP medium as $k$ times the real elastic constants $S_{i j k l}$,

$$
S_{i j k l}^{\prime}=k S_{i j k l} \text {. }
$$

We set the parameter $k$, simulating the mismatch between the media, within the range of $0.95<k<1.15$. Note that this interval corresponds to a mismatch of the wave velocity ranging between $-2.5 \%$ and $+7 \%$. $k=1$ means that the simulation medium matches perfectly the real one. This choice in implementing the medium mismatch is a simplification. Adopting the same deviation from actual values for all the elastic constants corresponds to (a) having similar error probability distribution for each elastic constant, the difference, for example, just regarding the mean value, and (b) having the same deviation from the actual value in sampling each of them. Both of these conditions may not be generally matched in laboratory measurements. However, this simplification allows one to investigate the first order effects of medium mismatch using a single control parameter, $k$.

The temporal retrofocalization of the wavefield is analyzed in Fig. 4 (left column), for $n=53$. From the top to the bottom, we considered these cases: $\Delta=0.6 \mathrm{~ms}, k=1 ; \Delta$ 

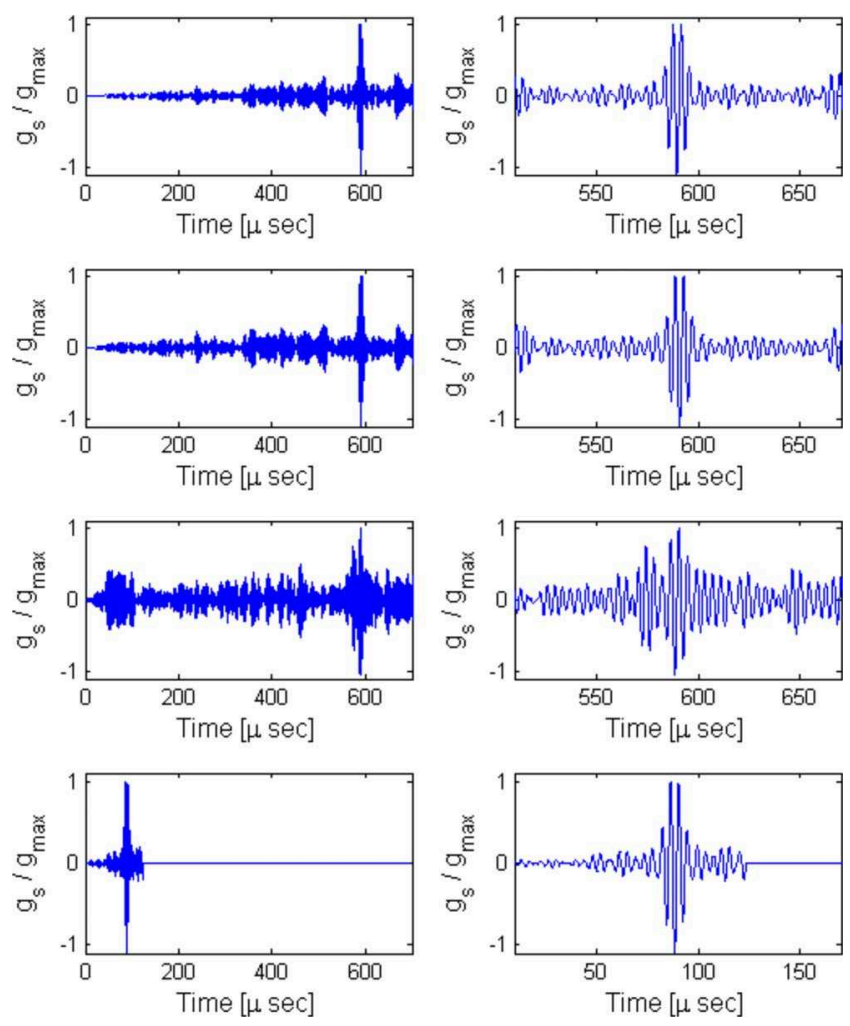

FIG. 4. (Color online) Temporal focusing of the time reversed signal at the source position when the backpropagation medium does not correspond to the real medium. Here, we used $n=53$ receivers. When a long coda is used, retrofocalization is good for $k=1$ and $k=0.992$ (upper two rows), but not for $k=1.05$ (third row). Using a shorter inverted signal (short coda), the quality of retrofocalization for $k=1.05$ is better (last row). The time signals at the source position are normalized to 1 . See also Fig. 5.

$=0.6 \mathrm{~ms}, k=0.992 ; \Delta=0.6 \mathrm{~ms}, k=1.05 ;$ and $\Delta=0.1 \mathrm{~ms}, k$ $=1.05$. In the first three rows, we observe that increasing the error in the estimation of the elastic constants, the quality of the focusing becomes progressively poorer. While the case $k=0.992$ (second row) gives approximately the same results as the ideal case ( $k=1$, first row), the source signal is not at all reconstructed already for $k=1.05$, i.e., for an error on the velocity estimation of only $2.5 \%$. The results are even worst for larger values of $k$ when the TR procedure fails even if a short coda is used (results not reported in this work for brevity). However, as shown in the last row of Fig. 4, an improvement in the retrofocalization (for the same value of $k$, $k=1.05)$ is obtained when a short coda is used. This is a direct result of the extreme sensitiveness of multipathing regimes to medium mismatch; ${ }^{38}$ meaning, the cumulative error is decreased using less coda, thereby improving the result. The spatial focusing supports the conclusions. The results are reported in Fig. 5.

To quantify the robustness of the TR procedure as a function of $n$ and $\Delta$, we define temporal and spatial retrofocusing evaluation metrics,

$$
\begin{aligned}
& \alpha=\max \left[g_{s}(t)\right] /\left\langle\left|g_{s}(t)\right|\right\rangle, \\
& \beta=\max [M] /\langle M\rangle .
\end{aligned}
$$

The maximum and mean $(\langle\cdot\rangle)$ values for the definition of the temporal indicator $\alpha$ are calculated in an interval around the
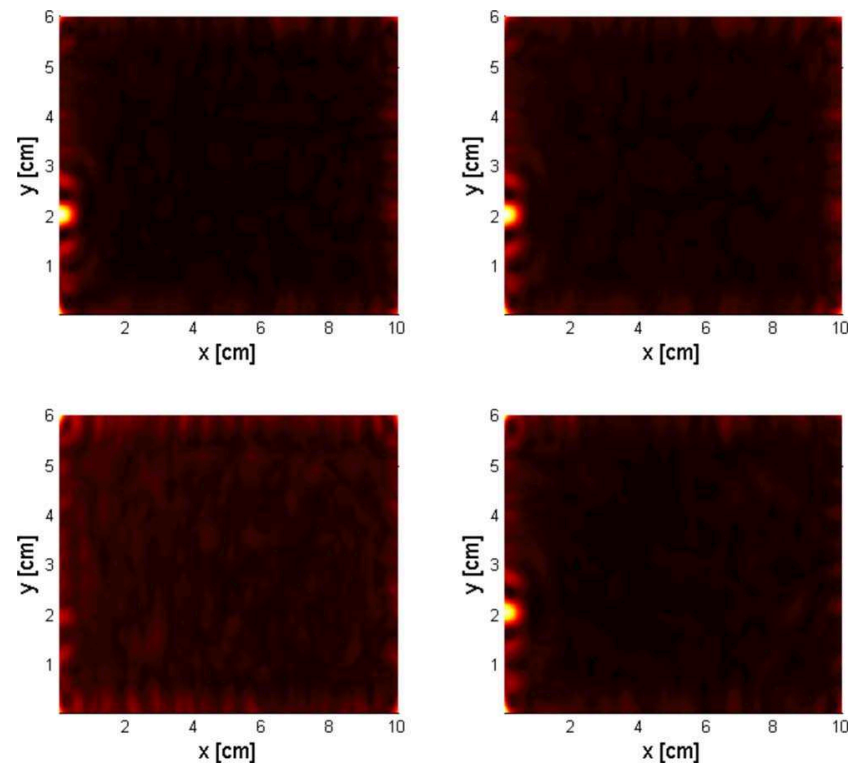

FIG. 5. (Color online) Spatial focusing of the time reversed signal at the source position when the backpropagation medium does not correspond to the real medium (same case as in Fig. 4). Here, we used $n=53$ receivers. When a long coda is used, retrofocalization is good for $k=1$ and $k=0.992$ (left and right plots in the top row), but not for $k=1.05$ (left plot in the bottom row). Still for $k=1.05$, using a shorter inverted signal (short coda), the quality of retrofocalization is better (right plot of the second row).

focal time: $0.5 \Delta<t<1.2 \Delta$ (the results are qualitatively independent of the choice of this interval). The maximum and mean values for the spatial indicator, $\beta$, are calculated on the entire sample. Note that the temporal indicator gives only information about temporal compression of the signal and not on the quality of the signal reconstruction, as will be discussed in Sec. III B (see comments regarding Fig. 13).

The values of $\alpha$ and $\beta$ are reported versus $k$ for the three lengths of the time window in Figs. $6-8 \quad(n=53, n=20, n$ $=7$, respectively). The results, independent of the values of $n$, indicate that

- the TR procedure is not robust when the TRBP modeled medium does not match well the real medium. When $k>1.1$ (5\% error in the propagation velocities), independent of $\Delta$, the metrics (both temporal and spatial) fall within the noise level (indicated as a dotted black line and defined in the caption of Fig. 6).

- The robustness is increased when the time window is shorter. However, that results in a reduction in the peak quality (value of the indicator for $k=1$ ), at least for the temporal metric.

\section{B. Enhancing robustness}

The results reported in Sec. III A indicate that computational TR imaging is not sufficiently robust. Indeed, the signals recorded during the backward propagation at the original source position are, in the case $k=1$ (no medium mismatch), almost one order of magnitude larger than the signals obtained with other values of $k$, i.e., when focusing is not occurring (either not perfectly or not at all). However, as 

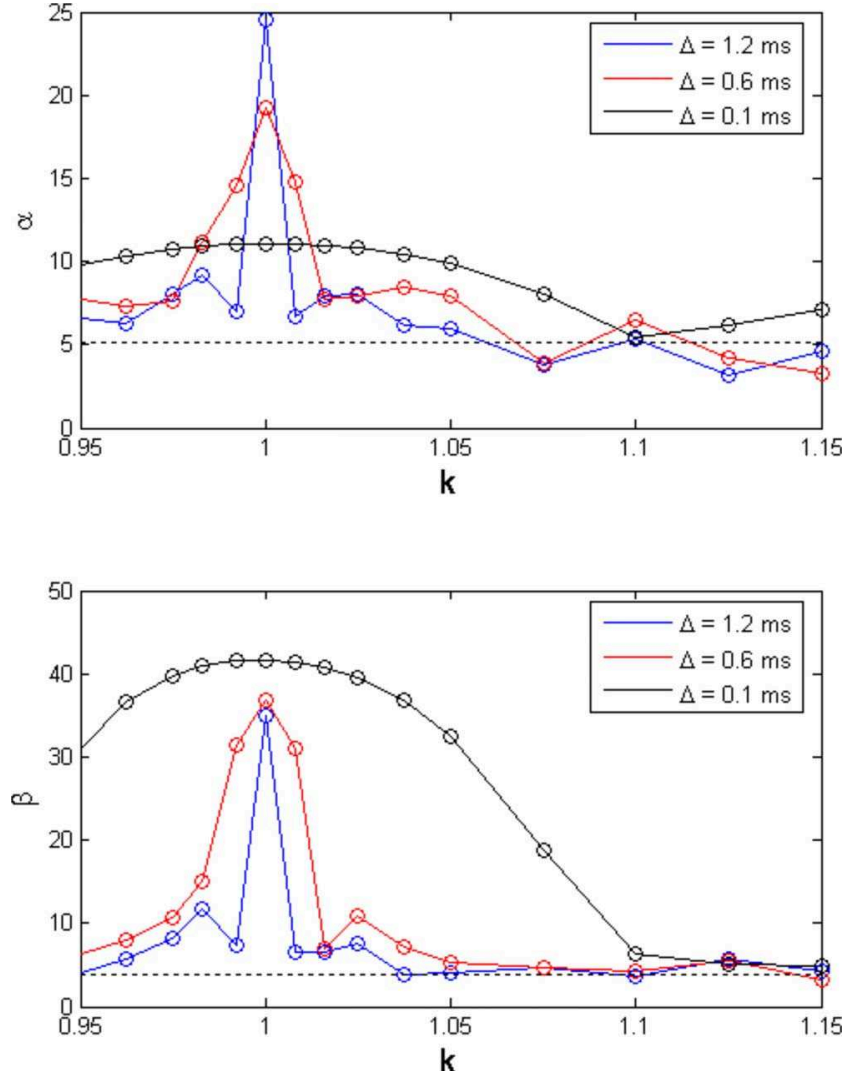

FIG. 6. (Color online) Temporal (upper plot) and spatial (lower plot) metrics as a function of $k$, for a choice of long, medium, and short coda of the inverted temporal signals. Case with a large number of receivers: $n=53$. For the definitions see Eqs. (4) and (5). The dotted lines in the plots represent the noise level: the noise temporal indicator level is defined as the metric calculated on a signal recorded far from the focal point; the noise spatial indicator level is calculated excluding from the mean and maxima calculations the focal region of the matrix $M$.

we show in this work, a significant improvement in the robustness can be obtained. In this subsection, we consider everywhere $n=53$ and $\Delta=0.6 \mathrm{~ms}$.

First of all, we note that time compressing/stretching the signals $u_{i}^{\text {rev }}(t)$, before using them as sources for the backward propagation, corresponds to "artificially" create experimental signals obtained as if the real medium had different elastic constants, all rescaled by the same factor, given by the square of the compression/stretching parameter.

Starting from these observations, we can define a new time-reversed signal at each receiver $i, \widetilde{u}_{i}^{\text {rev }}(t)$, starting from the one measured during the FP, $u_{i}^{\text {rev }}(t)$. This new signal is constructed as the linear superposition of its original version and of other signals obtained from it by time scaling. The time scaling consists of both a compression and a dilation part,

$$
\widetilde{u}_{i}^{\mathrm{rev}}(t)=u_{i}^{\mathrm{rev}}(t)+\sum_{j=1}^{Z}\left\{u_{i}^{\mathrm{rev}}\left[\left(1-\gamma_{j}\right) t\right]+u_{i}^{\mathrm{rev}}\left[\left(1+\gamma_{j}\right) t\right]\right\}
$$

where $\left(1 \pm \gamma_{j}\right)$ are the time scaling parameters and $Z$ is the total number of different time scaling used. The back propagation is then performed using the $\widetilde{u}_{i}^{\mathrm{rev}}(t)$ as sources at the receivers positions. Equation (6) can be applied to experimentally measured signals as well but, in such case, it may
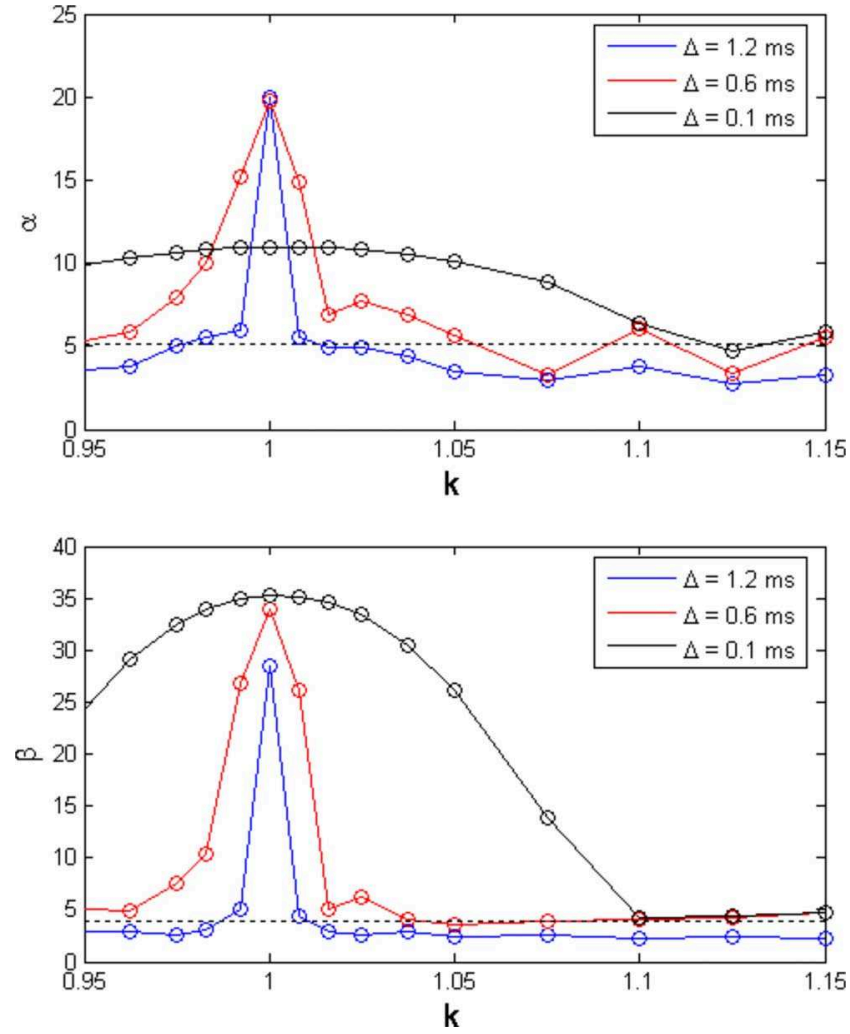

FIG. 7. (Color online) Temporal (upper plot) and spatial (lower plot) metrics as a function of $k$, for a choice of long, medium, and short coda of the inverted temporal signals. Case with an intermediate number of receivers: $n=20$. See also Fig. 6 .

be necessary to oversample $u_{i}^{\mathrm{rev}}(t), \forall i=1, \ldots, n$, before their temporal rescaling.

In Eq. (6), each term of the sum over the index $j$ takes into account the effect of a certain degree of elastic constants mismatch, being it either overestimation $\left[\left(1+\gamma_{j}\right)\right.$ time scaling factor] or underestimation $\left[\left(1-\gamma_{j}\right)\right]$. Rebroadcasting these new signals as sources for the backward propagation, we increase the probability of obtaining TR focusing: some of the compressed/stretched components of each new signal more likely contribute to the constructive interference leading to TR focusing than the simple signal $u_{i}^{\text {rev }}(t)$.

An example of the results obtained with $\widetilde{u}_{i}^{\text {rev }}(t)$ is reported in Fig. 9 for $Z=1$ and a time compression/dilation of the signal of $10 \%\left(\gamma_{1}=0.1\right)$. The temporal focus at the source when $k=1$ is almost the same using the standard approach (first row: $Z=0$ ) or using the superposition of three inverted signals (second row: $Z=1, \gamma_{1}=0.1$ ), thus showing that the use of signals modified using Eq. (6) does not destroy the retrofocalization. On the contrary, for $k=0.975$, the standard approach (third row) fails to obtain excellent focalization, while the approach proposed here provides a satisfactory result (last row). The same is valid also for spatial focusing (Fig. 10).

Figure 11 shows the temporal and spatial evaluation metrics for $Z=0, Z=1$, and $Z=2$, with $\gamma_{j}=0.05 j, \forall j=1,2$. Both $\alpha$ and $\beta$ still fall below the noise level for large errors in the elastic constant estimation. However, the robustness of the approach increases with $Z$, along with, as expected, a reduction in the indicator in the case $k=1$. 

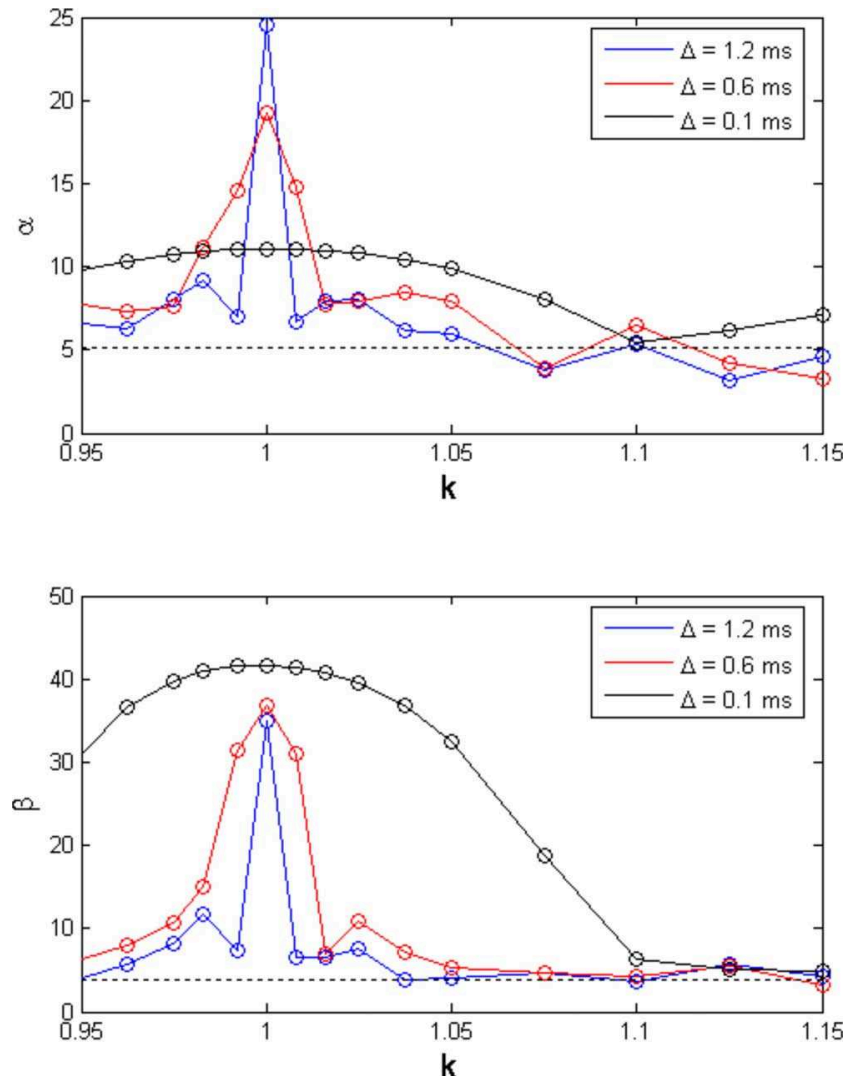

FIG. 8. (Color online) Temporal (upper plot) and spatial (lower plot) metrics as a function of $k$, for a choice of long, medium, and short coda of the inverted temporal signals. Case with a small number of receivers: $n=7$. See also Fig. 6.

Finally, in Fig. 12 we analyze the evolution of the temporal (upper plot) and spatial (lower plot) metrics as a function of $Z$ for different values of $k$. As expected, for $k=1$, the indicators remain very good for each value of $Z$. Indeed, the spatial metric decreases slightly for increasing $Z$, as expected. This is not the case for the temporal metric, which increases with $Z$, remaining always very high, thus indicating good focus. As already remarked, $\alpha$ provides information about temporal compression and not about the reconstruction of the source signal. Indeed, in Fig. 13 the signals recorded at the source position for $k=1$ are shown for $Z=0$ (upper plot) and $Z=2$ (lower plot). As visible, even though the metric is different for the two cases, the temporal compression is almost the same. The signal seems somewhat better reconstructed for $Z=0$ (for which the temporal metric is lower): compare the plots in the right column with the plot in Fig. 1(b). Therefore, we can say that changes in the temporal evaluation metric for $k=1$ are to be considered as negligible.

On the contrary, for $k>1$, Fig. 12 shows an improvement in both temporal and spatial metrics with increasing $Z$. A plateau is reached, where the metrics are almost independent from $Z$, as for the $k=1$ case. Exception is the case $k$ $=1.15$, where the corrections introduced are not sufficient to provide retrofocus. Finally, we point out that a tendency to a reduction in the metrics for larger $Z$ suggests the existence of a limit $Z$ value for optimization of the procedure. This limit will not further be investigated, being dependent on the configuration adopted.
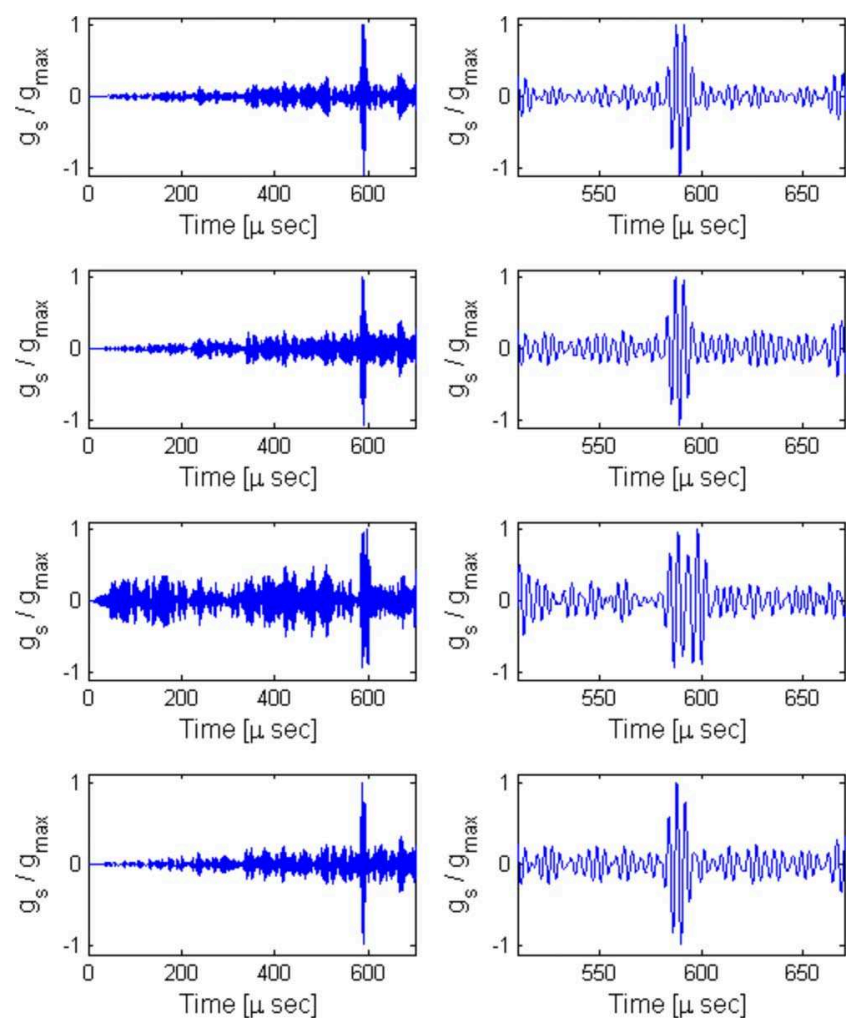

FIG. 9. (Color online) Temporal focusing of the time reversed signal on the source position when the correction on the reversal signals described by Eq. (6) is used, with $Z=1$ and $\gamma_{1}=0.1$. When $k=1$, there is almost no difference between the results using the standard approach $(Z=0$, upper row) or the corrected signals $(Z=1$, second row). When $k=0.975$, while the standard approach fails (third row), the correction proposed here makes the computational TRA imaging sufficiently robust (last row). The time signals at the source position are normalised to 1 . See also Fig. 10.
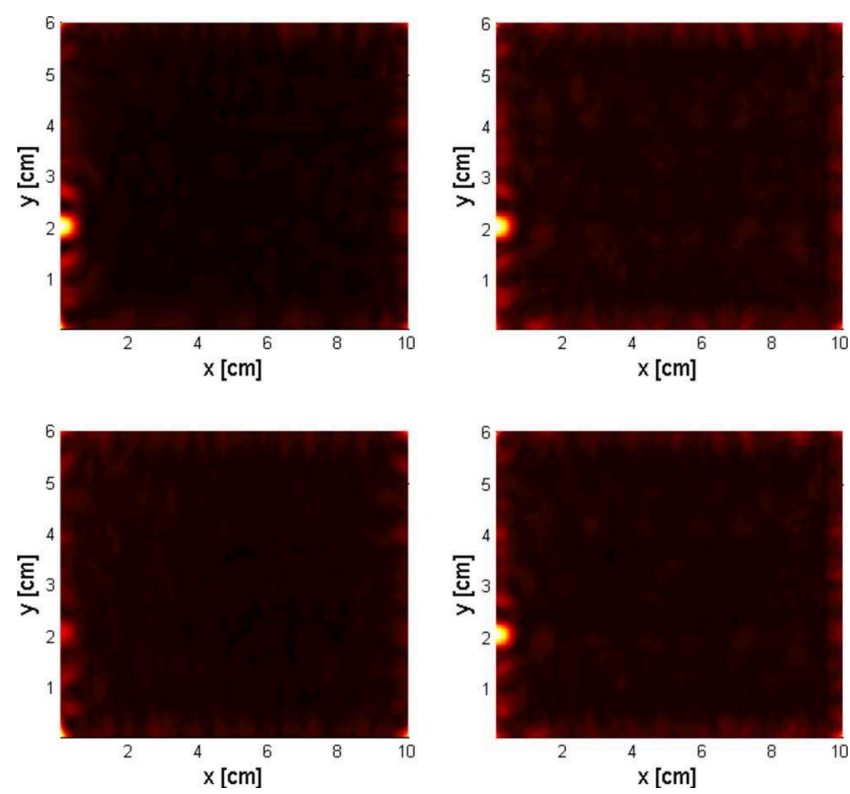

FIG. 10. (Color online) Spatial focusing of the time reversed signal on the source position when the correction on the reversal signals described by Eq. (6) is used, with $Z=1$ and $\gamma_{1}=0.1$. When $k=1$ (upper row), there is almost no difference between the results using the standard approach $(Z=0$, left) or the corrected signals $(Z=1$, right). When $k=0.975$ (second row), while the standard approach fails (left), the correction proposed here makes the computational TRA imaging sufficiently robust (right). 

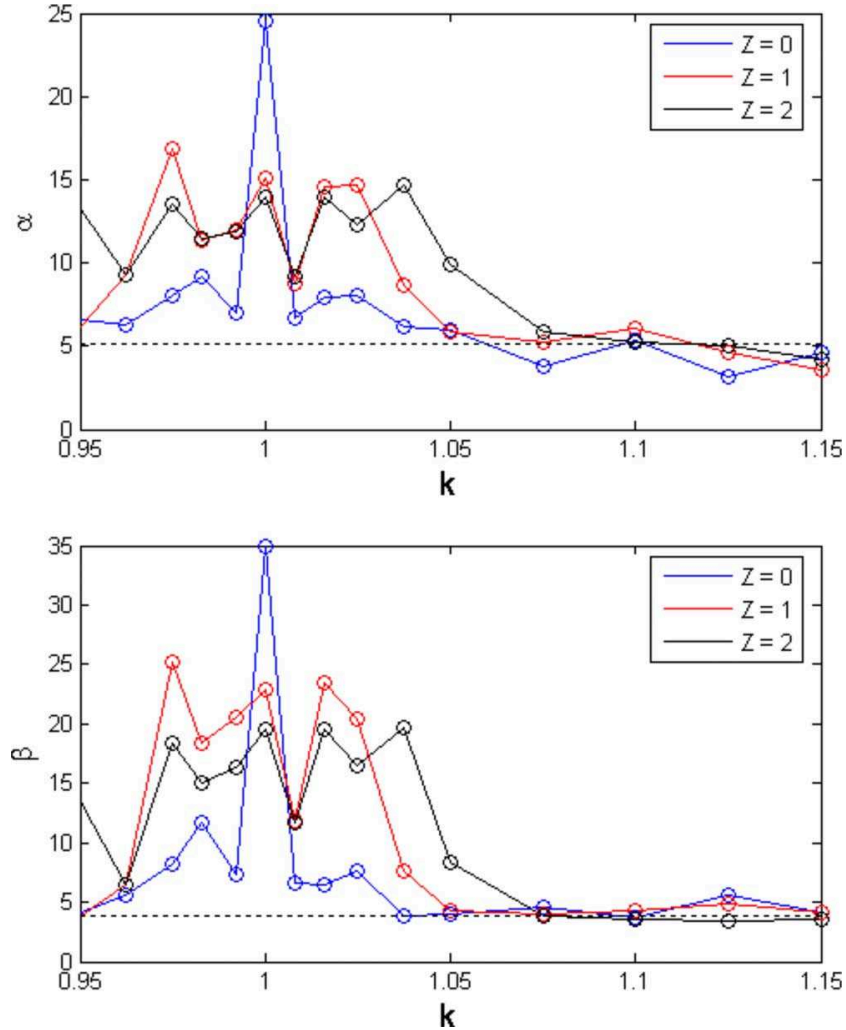

FIG. 11. (Color online) Temporal (upper plot) and spatial (lower plot) metrics as a function of $k$ for $n=53$ and $\Delta=0.6 \mathrm{~ms}$. The curves show that increasing the number $Z$ of signals used to correct the inverted signals to be backpropagated [see Eq. (6)] the robustness of the computational TRA imaging is improved.

\section{ROBUSTNESS AND HETEROGENEITY OF THE MEDIUM}

The second approximation usually performed in the ideal medium is that it is often assumed homogeneous, while that is not necessarily the case for the real medium, which may be a random medium or it might contain small hidden inclusions. To investigate the robustness of computational TR imaging in the case of heterogeneity of the real medium, we modified the case study discussed above. In particular, we performed new numerical simulations of the FP stage in order to provide different sets of input signals for the following three cases:

- random medium, i.e., the elastic constants of the aluminum plate are considered to be randomly distributed about the exact values given in Sec. II A. We chose a white noise distribution with zero average and $20 \%$ strength, i.e., elastic constants that differ at most of $20 \%$ from the exact values;

- soft inclusion: we placed a small square inclusion, centered at $(7.0,3.0) \mathrm{cm}$ and with $0.5 \mathrm{~cm}$ side, with elastic constants 0.2 times the original values; and

- hard inclusion: a small square inclusion with elastic constants five times the original ones, centered at $(7.0,3.0) \mathrm{cm}$ with $0.5 \mathrm{~cm}$ side.

The three sets of time signals obtained for the three cases were time reversed and each sent back in an "ideal" homogeneous medium with exact elastic constants $(k=1)$. For the
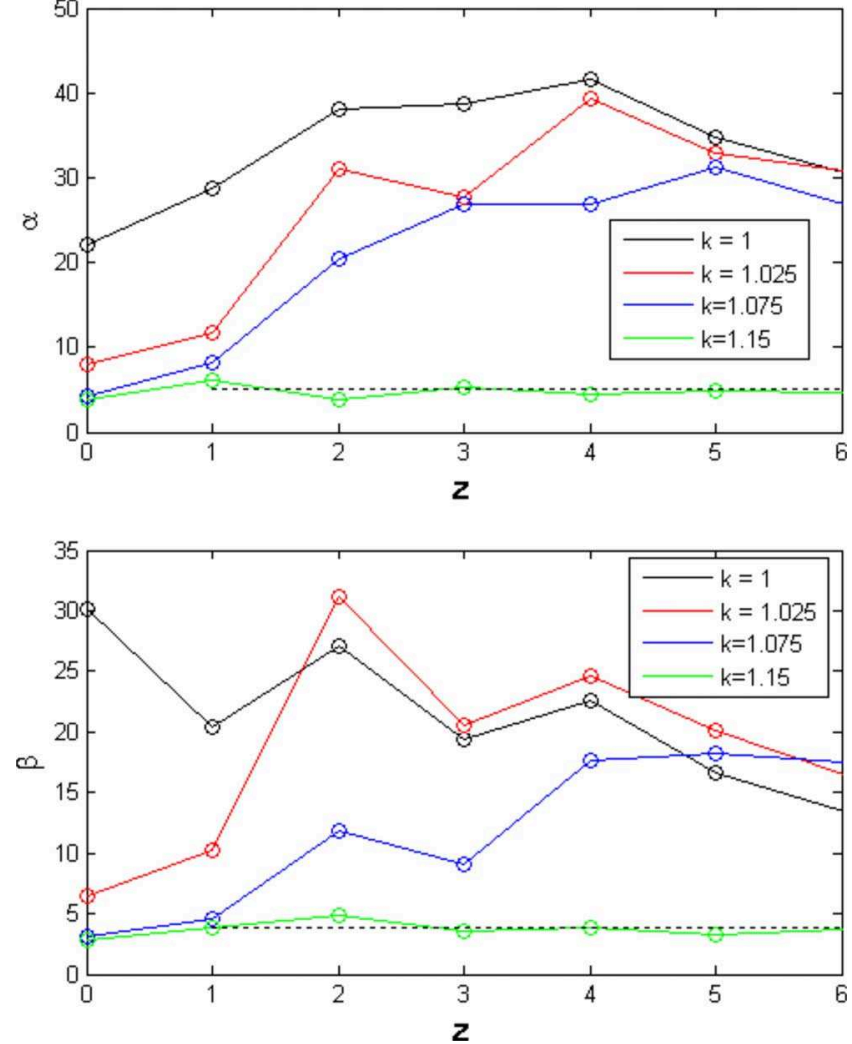

FIG. 12. (Color online) Temporal (upper plot) and spatial (lower plot) metrics as a function of $Z$ for $n=53$ and $\Delta=0.6 \mathrm{~ms}$. Here, $\gamma_{j}=0.05 j$.

TRBP, we used $n=53$ and $\Delta=0.6 \mathrm{~ms}$. The results of the simulations are reported in Fig. 14: random medium, soft, and hard inclusions, from the top to the bottom, respectively. In all cases, temporal compression, signal shape reconstruction, and spatial focusing are excellent. This is to be expected since the inclusion is small and most of the paths from the source to the receivers are not much influenced by its presence. Based on our findings, larger inclusions (or impedance mismatch) would be expected to affect the robustness of the computational TRA imaging method.

The influence of nonlinear scatterers, e.g., cracks, located by TR imaging methods combined with nonlinear elastic wave spectroscopy techniques ${ }^{10,12-14}$ is not analyzed in this work. However, as shown by Gliozzi et al. ${ }^{12}$ we do not
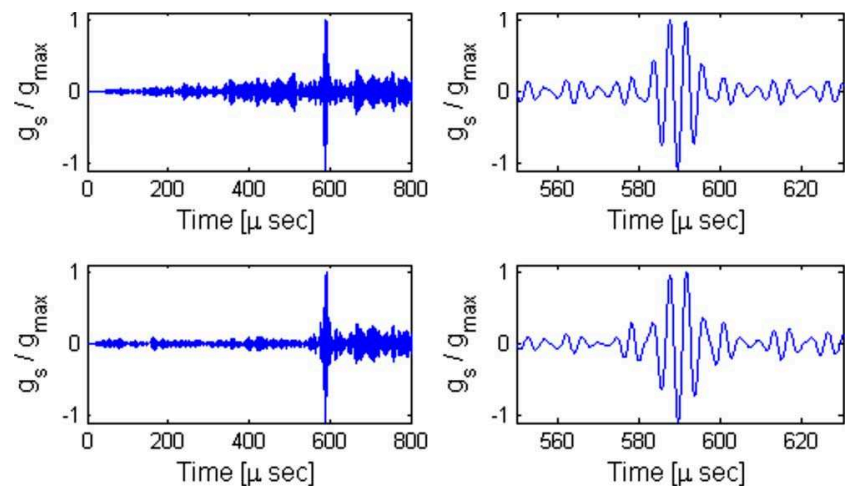

FIG. 13. (Color online) Temporal signals at the source position for $n=53$, $D=0.6 \mathrm{~ms}$, and $k=1$. Upper plot: $Z=0$; lower plot: $Z=2$. The right column represents a zoom around focal time. Signals are normalized to one. 

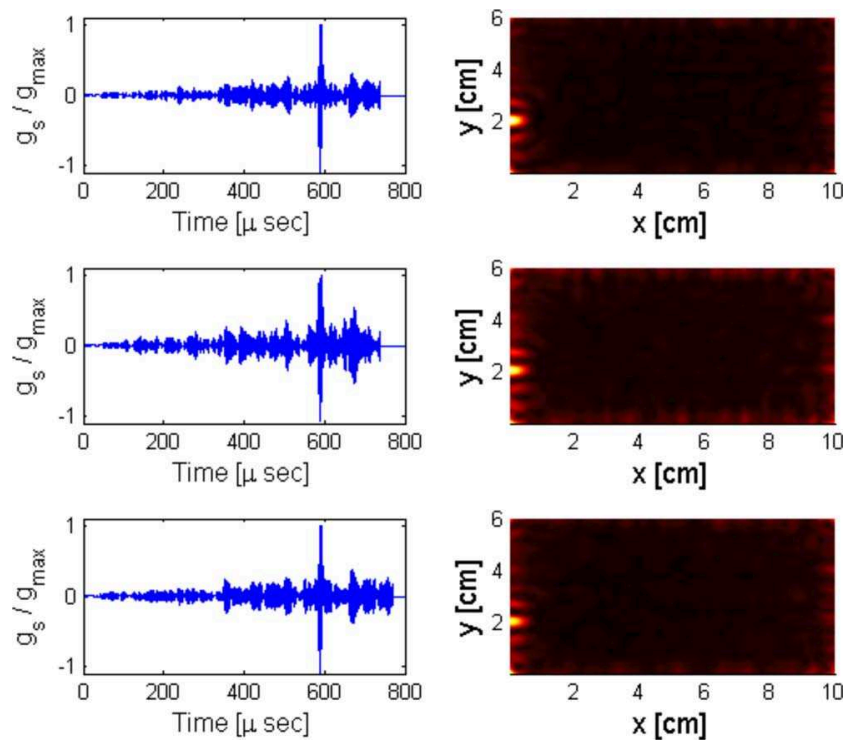

FIG. 14. (Color online) Temporal focusing of the time reversed signal on the source position and spatial focusing in the case of a heterogeneous real propagation medium. Upper row: random medium; middle row: soft inclusion; and lower row: hard inclusion.

expect such nonlinear scatterers to affect significantly the robust TR imaging procedure presented here, unless the scatterers themselves are very large.

\section{CONCLUSIONS}

In this paper, we have analyzed the robustness of a TRAbased computational approach to locating a source/scatterer. According to this approach, the TRBP of some experimentally measured signals is performed numerically. In this work, these "experimental" signals are generated via numerical simulations as well. We have investigated the effects due to uncertainties in the elastic constants of the propagation medium leading to a mismatch between the real FP medium and the one modeled for the simulated back propagation. We have shown that this TR approach to locating a source/ scatterer is not robust with respect to under/over estimation of the elastic wave velocities in the sample. Already uncertainties of about $2 \%$ are sufficient to destroy the retrofocalization at the source of the elastic wavefields.

We have shown that when the velocity fields are not well characterized, it is better to use small portions of the FP signals for the TRP. However, that is not always optimal from the experimental point of view since the use of a short TR interval has to be compensated for by the use of a large number of receivers.

Our implementation of the elastic constants mismatch between the forward and backward propagation's media assumes an identical relative difference for each elastic constant. Future work should address the role of different degrees of mismatch in the distinct elastic constants. Indeed, a larger deviation in the estimate of one elastic constant compared to the others can perturb significantly the scattering angles at interfaces. In the case of highly reverberant or multiply scattering media, this change in scattering angles magnifies the destruction of the TR focusing.
We have also proposed a method aiming at improving the robustness of the imaging procedure by using the superposition of properly time-scaled FP signals. This method exploits in a different way the same principle at the basis of the approach of Liu et al., ${ }^{29,39}$ i.e., sampling of the medium's elastic response from an ensamble of realizations of the medium itself. However, in our case the time scaling of the FP signals is equivalent to obtaining the respective signals with media having different elastic constansts under the assumption that all the elastic constants have been rescaled by the same amount. A single FP is sufficient instead of several. The technique of Liu et al. applies specifically to random media and requires performing several FP experiments in order to measure different Green's functions in correspondence to different realizations of the medium itself. The efficiency of our method is constrained by the assumption of identical relative errors for all the elastic constants. Although this condition may not be achieved in some experiments, the approach presented in this work can still be adopted with some modifications. For instance, a possible solution could consist in performing several back propagation simulations using the strectching/dilation processing on the FP signals as suggested here but varying, from one simulation to the other, the scaling parameter $k$ for the elastic constants. In addition, the method proposed in this article can be very useful in several realistic cases, for example, when the sample undergoes perturbations that change all of its elastic properties of the same relative amount, e.g., temperature fluctuations in time. ${ }^{27,28}$

Finally, we have shown that the computational TRA imaging method is very robust in the presence of the heterogeneities of the real medium. Random fluctuations in the elastic constants (not measurable) or the presence of small (soft or hard) heterogeneities, not included in the model of the medium for the TRBP because it is unknown, does not affect much the reconstruction process.

The results reported in this work prove, in our opinion, the feasibility of numerical backpropagation of real experimental data, also when the medium properties cannot be accurately well estimated. The effects due to the boundary conditions of the sample are currently under investigation and application to real data is planned for the future.

\section{ACKNOWLEDGMENTS}

This work has been funded by Institutional Support (LDRD Program) at the Los Alamos National Laboratory. We thank Dr. A. S. Gliozzi (Politecnico di Torino, Italy) and R. A. Guyer, C. Larmat, B. E. Anderson, P.-Y. Le Bas, and T. J. Ulrich (Los Alamos National Laboratory) for useful comments and discussions. One of us (M.S.) would like to thank the Los Alamos National Laboratory for financial support.

${ }^{1}$ M. Fink, D. Cassereau, A. Derode, C. Prada, P. Roux, M. Tanter, J. L. Thomas, and F. Wu, Rep. Prog. Phys. 63, 1933 (2000).

${ }^{2}$ M. Fink, Phys. Today 50(3), 34 (1997).

${ }^{3}$ B. E. Anderson, M. Griffa, C. Larmat, T. J. Ulrich, and P. A. Johnson, Acoust. Today 4(1), 5 (2008).

${ }^{4}$ M. Tanter, J.-L. Thomas, and M. Fink, J. Acoust. Soc. Am. 108, 223 (2000).

${ }^{5}$ C. Draeger and M. Fink, Phys. Rev. Lett. 79, 407 (1997).

${ }^{6}$ J. F. Claerbout, Geophysics 36, 467 (1971). 
${ }^{7}$ W.-F. Chang and G. A. McMechan, Geophysics 52, 1365 (1987).

${ }^{8}$ E. Kerbrat, C. Prada, D. Cassereau, and M. Fink, IEEE Trans. Ultrason Ferroelectr. Freq. Control 49, 1103 (2002).

${ }^{9}$ G. Montaldo, D. Palacio, M. Tanter, and M. Fink, IEEE Trans. Ultrason. Ferroelectr. Freq. Control 52, 1489 (2005).

${ }^{10}$ A. M. Sutin and P. A. Johnson, AIP Conf. Proc. 760, (1), 385 (2005).

${ }^{11}$ T. J. Ulrich, P. A. Johnson, and A. Sutin, J. Acoust. Soc. Am. 119, 1514 (2006).

${ }^{12}$ A. S. Gliozzi, M. Griffa, and M. Scalerandi, J. Acoust. Soc. Am. 120, 2506 (2006).

${ }^{13}$ G. Zumpano and M. Meo, Int. J. Solids Struct. 44, 3666 (2007).

${ }^{14}$ T. J. Ulrich, P. A. Johnson, and R. A. Guyer, Phys. Rev. Lett. 98, 104301 (2007).

${ }^{15}$ C. Larmat, J.-P. Montagner, M. Fink, Y. Capdeville, E. Clévédé, and A. Tourin, Geophys. Res. Lett. 33, L19312 (2006).

${ }^{16}$ C. Larmat, J. Tromp, Q. Liu, and J.-P. Montagner, J. Geophys. Res. 113, B09314 (2008).

${ }^{17}$ W. A. Kuperman, W. S. Hodgkiss, H. C. Song, T. Akal, C. Ferla, and D. R Jackson, J. Acoust. Soc. Am. 103, 25 (1998).

${ }^{18}$ H. Tortel, G. Micolau, and M. Saillaud, J. Electromagn. Waves Appl. 13, 687 (1999).

${ }^{19}$ D. Liu, G. Kang, L. Ling, Y. Chen, S. Vasudevan, W. Joines, Q. H. Liu, J. Krolik, and L. Carin, IEEE Trans. Antennas Propag. 53, 3058 (2005).

${ }^{20}$ S. K. Lehman and A. J. Devaney, J. Acoust. Soc. Am. 113, 2742 (2003).

${ }^{21}$ M. Scalerandi, A. S. Gliozzi, B. E. Anderson, M. Griffa, P. A. Johnson, and T. J. Ulrich, J. Phys. D: Appl. Phys. 41, 155504 (2008).

${ }^{22}$ B. E. Anderson, T. J. Ulrich, M. Griffa, P.-Y Le Bas, M. Scalerandi, A. S. Gliozzi, and P. A. Johnson J. Appl. Phys. 105, 083506 (2009).
${ }^{23}$ L. Borcea, G. Papanicolaou, C. Tsogka, and J. Berryman, Inverse Probl. 18, 1247 (2002).

${ }^{24}$ J. M. Carcione, Wave Fields in Real Media. Wave Propagation in Anisotropic, Anelastic, Porous and Electromagnetic Media (Elsevier, New York, 2007), Vol. 38

${ }^{25}$ M. Scalerandi, A. S. Gliozzi, C. L. E. Bruno, and K. Van Den Abeele, J. Phys. D: Appl. Phys. 41, 215404 (2008).

${ }^{26}$ A. Derode, P. Roux, and M. Fink, Phys. Rev. Lett. 75, 4206 (1995).

${ }^{27}$ A. Tourin, A. Derode, and M. Fink, Phys. Rev. Lett. 87, 274301 (2001).

${ }^{28}$ S. Kim W. A. Kuperman, W. S. Hodgkiss, H. C. Song, G. F. Edelmann, and T. Akal, J. Acoust. Soc. Am. 114, 145 (2003).

${ }^{29}$ D. Liu S. Vasudevan, J. Krolik, G. Bal, and L. Carin, IEEE Trans. Antennas Propag. 55, 344 (2007).

${ }^{30} \mathrm{G}$. Bal and R. Verastegui, Multiscale Model. Simul. 2, 639 (2004).

${ }^{31}$ R. Snieder, A. Grêt, H. Douma, and J. Scales, Science 295, 2253 (2002).

${ }^{32}$ G. Shan, L. Zhang, Y. Wang, T. Nemeth, and W. Liu, SEG Exp. Abstr. 27, 2321 (2008).

${ }^{33}$ P. Sava and B. Biondi, Geophys. Prospect. 52, 593 (2004).

${ }^{34}$ P. P. Delsanto and M. Scalerandi, Phys. Rev. B 68(6), 064107 (2003).

${ }^{35}$ R. D. Huber, R. B. Mignogna, K. E. Simmonds, R. S. Schechter, and P. P. Delsanto, Ultrasonics 35, 7 (1997).

${ }^{36}$ M. Bentahar, H. El Aqra, R. El Guerjouma, M. Griffa, and M. Scalerandi, Phys. Rev. B 73(1), 014116 (2006).

${ }^{37}$ P. P. Delsanto, P. A. Johnson, M. Scalerandi, and J. A. TenCate, J. Phys. D: Appl. Phys. 35, 3145 (2002).

${ }^{38}$ R. K. Snieder and J. A. Scales, Phys. Rev. E 58, 5668 (1998).

${ }^{39}$ D. Liu, J. Krolik, and L. Carin, IEEE Trans. Geosci. Remote Sens. 45, 934 (2007). 